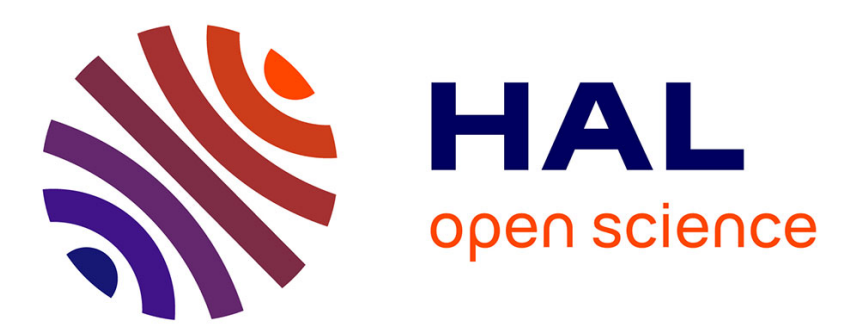

\title{
Test of GNSS Receiver Behavior in Presence of Multiple Correlation Peaks Induced by Evil Waveform
}

\author{
Ikhlas Selmi, Paul Thevenon, Christophe Macabiau, Jaron Samson
}

\section{To cite this version:}

Ikhlas Selmi, Paul Thevenon, Christophe Macabiau, Jaron Samson. Test of GNSS Receiver Behavior in Presence of Multiple Correlation Peaks Induced by Evil Waveform. 34th International Technical Meeting of the Satellite Division of The Institute of Navigation (ION GNSS+ 2021), Sep 2021, St. Louis, United States. pp.4183-4197, 10.33012/2021.18088 . hal-03407539

\section{HAL Id: hal-03407539 \\ https://hal-enac.archives-ouvertes.fr/hal-03407539}

Submitted on 28 Oct 2021

HAL is a multi-disciplinary open access archive for the deposit and dissemination of scientific research documents, whether they are published or not. The documents may come from teaching and research institutions in France or abroad, or from public or private research centers.
L'archive ouverte pluridisciplinaire HAL, est destinée au dépôt et à la diffusion de documents scientifiques de niveau recherche, publiés ou non, émanant des établissements d'enseignement et de recherche français ou étrangers, des laboratoires publics ou privés. 


\title{
Test of GNSS receiver behavior in presence of Multiple Correlation Peaks Induced by Evil Waveform
}

\author{
Ikhlas Selmi, ENAC, Université de Toulouse \\ Paul Thevenon, ENAC, Université de Toulouse \\ Christophe Macabiau, ENAC, Université de Toulouse \\ Jaron Samson, ESA
}

\section{Biography}

Ikhlas Selmi is currently part of the SIGnal processing and NAVigation (SIGNAV) research axis of TELECOM team at ENAC. She graduated as engineer in Telecommunication and Signal Processing from ESIEE. She received her Ph.D. in 2013 from Telecom SudParis on mitigating GNSS interference between indoor and outdoor signals. She is currently working on GNSS signal distortion generated by the satellite payload.

Paul Thevenon graduated as electronic engineer from Ecole Centrale de Lille in 2004 and obtained in 2007 a research master at ISAE in space telecommunications. In 2010, he obtained a PhD degree in the signal processing laboratory of ENAC in Toulouse, France. From 2010 to 2013, he was employed by CNES, the French space agency, to supervise GNSS research activities and measurement campaigns. Since the July 2013, he is employed by ENAC as Assistant Professor. His current activities are GNSS signal processing, GNSS integrity monitoring and hybridization of GNSS with other sensors.

Christophe Macabiau graduated as electronics engineer in 1992 from the ENAC in Toulouse, France. Since 1994, he has been working on the application of satellite navigation techniques to civil aviation. He received his Ph.D in 1997 and has been in charge of the signal processing lab of ENAC from 2000 to 2012 . He is currently the head of the TELECOM team of ENAC.

Jaron Samson works as a Navigation Engineer at ESA's Technical Centre (ESTEC), being involved in EGNOS, GNSS Evolutions, and R\&D related to navigation. From 2012 until 2021, Jaron has worked as a System Engineer at ESA's EGNOS Project Office in Toulouse (France). Jaron holds an M.Sc-degree in Physical and Mathematical Geodesy from Delft University of Technology (The Netherlands).

\begin{abstract}
Evil Waveforms (EWF) are non-nominal distortions that can be observed on satellite signals and cause additional bias on the estimated user position. A Threat Model (TM) has been proposed by ICAO for GPS L1 C/A to describe the possible distortions that can be observed on the GPS signals. This Threat model is also adopted for Galileo E1-C and E5a-Q signals. To evaluate the induced tracking bias by the EWFs of the ICAO TM, the receiver behavior after their occurrence needs to be assessed. In addition, some EWF cases induces multiple peaks on the correlation function. The main question of this study is: how the receiver deals with the multiple peaks situation and what impact it can have on the induced EWF bias. The present paper evaluates the effect of two Receiver Behavior Models (RBM) based on two possible reactions of the DLL after EWF occurrence: loss and no loss of the tracking. Those RBMs are modelled using different parameters such as the DLL pull in area and the C/NO loss. The study shows the occurrence of multiple
\end{abstract}


zero crossing situations and the EWF induced bias for both RBMs. The sensitivity of these results to the EWF Threat Space and tested User Space is then analyzed for both RBMs. This paper presents the results obtained when testing TM-A, B and C on Galileo E5a signal. Then, it discusses the observed impacts of the two tested RBMs and the situation of multiple zero crossing on the EWF induced tracking bias.

\section{INTRODUCTION}

In 1993, the first important GNSS signal distortion due to a payload failure was observed on GPS L1 C/A [1]. This event prompted the GNSS community to standardize a kind of non-nominal signal distortion, named Evil Waveforms (EWF). A Threat Model (TM) has been proposed by ICAO for GPS L1 C/A to describe the possible distortions that can be observed on GPS signals [2]. The goal of the TM was to study three possible effects on GNSS correlation function computed by a receiver: dead zones (flat spot in the correlation function), distortions (correlation function asymmetry) and false peaks. The focus of this paper is on a consequence of the later effect, and in particular, on how a receiver will behave in presence of multiple correlation peaks induced by the combination of an Evil Waveform and its RF and tracking characteristics. The present study will focus on the analysis of Galileo E5a results obtained for Dual Frequency Multi-constellation (DFMC) user receiver to assess the effect of multiple peaks on the correlation function (that translate to multiple zero crossings on the discriminator function). But similar results are obtained with DFMC User Space applied to GPS L1, L5, Galileo E1c.

The paper has the following structure. First, the EWF ICAO Threat Models and the considered assumptions when simulating them with the tested parameters of the DFMC User Space are presented in section 1. Then, an example of multiple zero crossing situation in presence of EWF and the reaction of the receiver in this scenario are analyzed. In section 3, the considered receiver behavior models in presence of EWF with multiple zero crossing and the associated parameters (such as the pull-in area and C/NO loss threshold) are defined. The analysis of the observed multiple zero crossing situations and the effect on the absolute tracking bias for Galileo E5a TM-A, B and C are given in section 4. In section 5 , the sensitivity of these effects to the User Space and Threat Space is analyzed. Finally, the conclusion will summarize the observed effects of the multiple zero crossing situation and the recommendations to deal with the possible integrity threat induced by this situation.

\section{SECTION 1 - DESCRIPTION OF THE EWF THREAT SPACE AND THE USER SPACE}

\section{EWF Threat Space}

ICAO proposed three types of failures that could be related to payload functions, to the observed EWF event of 1993, and that would result in at least one of the three problematic effects on GPS L1 C/A receivers [2]:

- $\quad$ Threat Model A (TM-A) consists of the normal C/A code except that all the positive chips have a falling edge that leads or lags relative to the correct end-time for that chip. This TM is associated with a failure in the navigation data unit (NDU), the digital partition of a GPS or GLONASS satellite

○ This type of failure results in the creation of a flat zone at the top of the correlation function and an offset on the entire correlation function.

- Threat Model B (TM-B) introduces amplitude modulation and models the degradations in the analog section of the GPS or GLONASS satellite. More specifically, it consists of the output from a second order system when the nominal C/A code (baseband signal) is the input. TM-B assumes that the degraded satellite subsystem can be described as a linear system dominated by a pair of complex conjugate poles. These poles are located at:

where $\quad \sigma \pm j 2 \pi f d$

- $\sigma$ is the damping factor in unit of Mnepers/s 
- $f_{d}$ is the resonant frequency in unit of $\mathrm{MHz}$.

- This type of failure results in the creation of distorted and false peaks.

- Threat Model C (TM-C) introduces both lead/lag and amplitude modulation. Specifically, it consists of outputs from a second order system when the C/A code signal at the input suffers from lead or lag. This waveform is a combination of the two effects described above.

These 3 threat models were endorsed by ICAO for GPS L1 C/A. As it can be understood, they depend upon 3 parameters:

- $\Delta$ representing the lead or lag relative to the correct end-time of the chip preceding the falling transition

- $\sigma$ and $f_{d}$ representing the second order system creating the amplitude modulation of the chip

The range of values of these parameters defines a so-called Treat Space (TS) that is representative of the feared events. The GPS L1 C/A TS is given in Table 1.

The proposed ICAO TSs for Galileo E1c and E5a signals are given in Table 2 [3].

Table 1. ICAO-standardized GPS L1 C/A (ICAO 2006) and L5 Threat Space

\begin{tabular}{|c|c|c|c|}
\hline ICAO parameters & $\Delta$ (Chips) & $\boldsymbol{\sigma}$ (Mnepers/s) & $\boldsymbol{f}_{\boldsymbol{d}}(\mathbf{M H z})$ \\
\hline TM-A & {$[-0.12-0.12]$} & - & - \\
\hline TM-B & - & {$[0.8-8.8]$} & {$[4-17]$} \\
\hline TM-C & {$[-0.12-0.12]$} & {$[0.8-8.8]$} & {$[7.3-13]$} \\
\hline
\end{tabular}

Table 2. ICAO Threat Space for Galileo E1C and E5a

\begin{tabular}{|c|c|c|c|c|}
\hline \multicolumn{2}{|c|}{ ICAO parameters } & $\Delta(\mu \boldsymbol{s})$ & $\sigma($ Mnepers/s) & $\boldsymbol{f}_{\boldsymbol{d}}(\mathrm{MHz})$ \\
\hline \multirow{2}{*}{ TM-A } & Galileo E1c & {$[-0.12-0.12]$} & - & - \\
\cline { 2 - 5 } & Galileo E5a & {$[-0.1-0.1]$} & & {$[0.1-18]$} \\
\hline \multirow{2}{*}{ TM-B } & Galileo E1c & - & {$[0.1-63]$} & {$[0.1-8]$} \\
\cline { 2 - 5 } & Galileo E5a & - & {$[0.1-23]$} & {$[0.1-18]$} \\
\hline \multirow{2}{*}{ TM-C } & Galileo E1c & {$[-0.12-0.12]$} & {$[0.1-63]$} & {$[0.1-8]$} \\
\cline { 2 - 5 } & Galileo E5a & {$[-0.1-0.1]$} & {$[0.1-23]$} & \\
\hline
\end{tabular}

User Space and considered assumption for EWF simulations

The considered receiver parameters at the reference station and the aircraft to simulate the EWF effect in the ENAC SW are given in Table 3.

A default assumption that is always considered in literature is that when the EWF appears, it is assumed that the signal instantaneously switches from nominal to an EWF status. An alternative assumption could be to consider the situation where the signal loses its nominal properties at $t_{\text {Failure }}$, then follows an unknown transient status and finally, takes on the EWF characteristics at $t_{E W F}\left(>t_{\text {Failure }}\right)$. In the case of this second assumption, there are more chances that the user/reference station receiver could notice the strange signal change (through C/NO variation and absence of significant useful signal leading to tracking loss). It seems that the first assumption may lead to worst scenario in term of integrity risk since the receiver may not detect the strange change in the signal caused by the presence of the EWF. The first assumption is considered in this study. It is also assumed that the smoothing filter has 
reached its steady state when the EWF occurs $\left(a t t_{E W F}\right)$. This assumption is usually considered in literature when studying EWF effects.

In addition, when processing EWF, the observed effect (on pseudorange measurements and correlation function shape) remains constant since its instant of occurrence $t_{E W F}$ and during the user and reference station process, notably during their smoothing filter convergence period. This assumption is also considered in this study and has an influence on the methodology used to determine the induced tracking error. The absolute tracking error due to the EWF is determined as the difference between the tracking error in presence of the EWF and the tracking error in nominal conditions. Both tracking errors are determined as the steady state bias (reached after filter convergence) associated with the zero crossing point of the DLL discriminator function. The EWF induced absolute and differential tracking errors are used to determine the probability of missed detection $\mathrm{P}_{\mathrm{md}}$ [4] to respect in order to satisfy the DFMC SBAS integrity risk requirements for each EWF. When the derived $\mathrm{P}_{\mathrm{md}}$ requirement is different from 1 , the processed EWF is considered as hazardous (can induce integrity risks). The worst EWF induced bias corresponds to the highest absolute bias which induces the lowest and most demanding $\mathrm{P}_{\mathrm{md}}$.

Note that, in the present study, the tracking errors are determined only in the presence of line-of-sight signal distorted by the EWF. The effect of Multipath (non line of sight signal) combined to EWF is not considered in this study.

Table 3. Parameters of Reference station and User Space applied to GPS L1/L5 and Galileo E1C/E5a for DFMC SBAS users

\begin{tabular}{|c|c|c|c|c|}
\hline & \multicolumn{2}{|c|}{$\begin{array}{c}\text { Galileo E1c signal (CBOC(6.1)) and GPS L1 } \\
\text { C/A }\end{array}$} & \multicolumn{2}{|c|}{ Galileo E5a and GPS L5 signal BPSK(10)) } \\
\hline & reference & user & reference & user \\
\hline $\begin{array}{l}\text { Tracking } \\
\text { technique }\end{array}$ & $\begin{array}{l}\text { EML (BOC(1.1) local } \\
\text { replica for Galileo } \\
\text { E1c) }\end{array}$ & $\begin{array}{l}\text { EML (BOC(1.1) local } \\
\text { replica for Galileo } \\
\text { E1c) }\end{array}$ & $\begin{array}{l}\text { EML }(B P S K(10) \\
\text { local replica) }\end{array}$ & $\begin{array}{l}\text { EML }(B P S K(10) \\
\text { local replica) }\end{array}$ \\
\hline $\begin{array}{c}\text { Correlator } \\
\text { spacing }\end{array}$ & \multicolumn{2}{|c|}{$0.08,0.1$ and 0.12 chip } & \multicolumn{2}{|c|}{$0.9,1$ and 1.1 chip } \\
\hline $\begin{array}{c}\text { Pre-correlation } \\
\text { bandwidth } \\
\text { (double-sided) }\end{array}$ & $24 \mathrm{MHz}$ & $\begin{array}{l}12,14,16,18,20 \\
22,24 \mathrm{MHz}\end{array}$ & $24 \mathrm{MHz}$ & $\begin{array}{l}12,14,16,18,20 \\
22,24 \mathrm{MHz}\end{array}$ \\
\hline $\begin{array}{l}\text { Equivalent } \\
\text { reception filter }\end{array}$ & $\begin{array}{l}\text { one filter is tested } \\
\text { (6th-order } \\
\text { Butterworth) to } \\
\text { estimate the } \\
\text { reference error as } \\
\text { the minimum on all } \\
\text { the correlator } \\
\text { spacing values }\end{array}$ & $\begin{array}{l}4 \text { filters are tested } \\
\text { (6th-order } \\
\text { Butterworth, 0-group } \\
\text { delay resonator, } 150 \\
\text { ns maximum } \\
\text { differential group } \\
\text { delay resonator, } 150 \\
\text { ns maximum } \\
\text { differential group } \\
\text { delay 6th-order } \\
\text { Butterworth) }\end{array}$ & $\begin{array}{l}\text { one filter is tested } \\
\text { (6th-order } \\
\text { Butterworth) to } \\
\text { estimate the } \\
\text { reference error as } \\
\text { the minimum on all } \\
\text { the correlator } \\
\text { spacing values }\end{array}$ & $\begin{array}{l}4 \text { filters are tested } \\
\text { (6th-order } \\
\text { Butterworth, 0- } \\
\text { group delay } \\
\text { resonator, } 150 \text { ns } \\
\text { maximum } \\
\text { differential group } \\
\text { delay resonator, } \\
150 \text { ns maximum } \\
\text { differential group } \\
\text { delay 6th-order } \\
\text { Butterworth) }\end{array}$ \\
\hline
\end{tabular}

\section{SECTION 2 - ILLUSTRATION OF MULTIPLE CORRELATION PEAKS AND THE RESULTING MULTIPLE ZERO CROSSING ON THE DISCRIMINATOR FUNCTION}

For some extreme ICAO threats where multiple peaks are visible as shown in Figure 1, the expected behavior of GNSS receiver may change depending on the used multiple peaks mitigation techniques. Figure 1 shows the correlation function on the left and the E-L discriminator (with an E/L chip spacing of 0.1 chip) on the right obtained 
when Galileo E1c is distorted by TM-C with $\Delta=0.09 \mu \mathrm{s}, \mathrm{F}_{\mathrm{d}}=11 \mathrm{MHz}$ and $\sigma=0.2 \mathrm{Mnepers} / \mathrm{s}$ and filtered with a $6^{\text {th }}$ order Butterworth filter at a bandwidth of $24 \mathrm{MHz}$. The obtained correlation function has multiple peaks corresponding to multiple zero crossings on the E-L discriminator that can be tracked by the receiver.
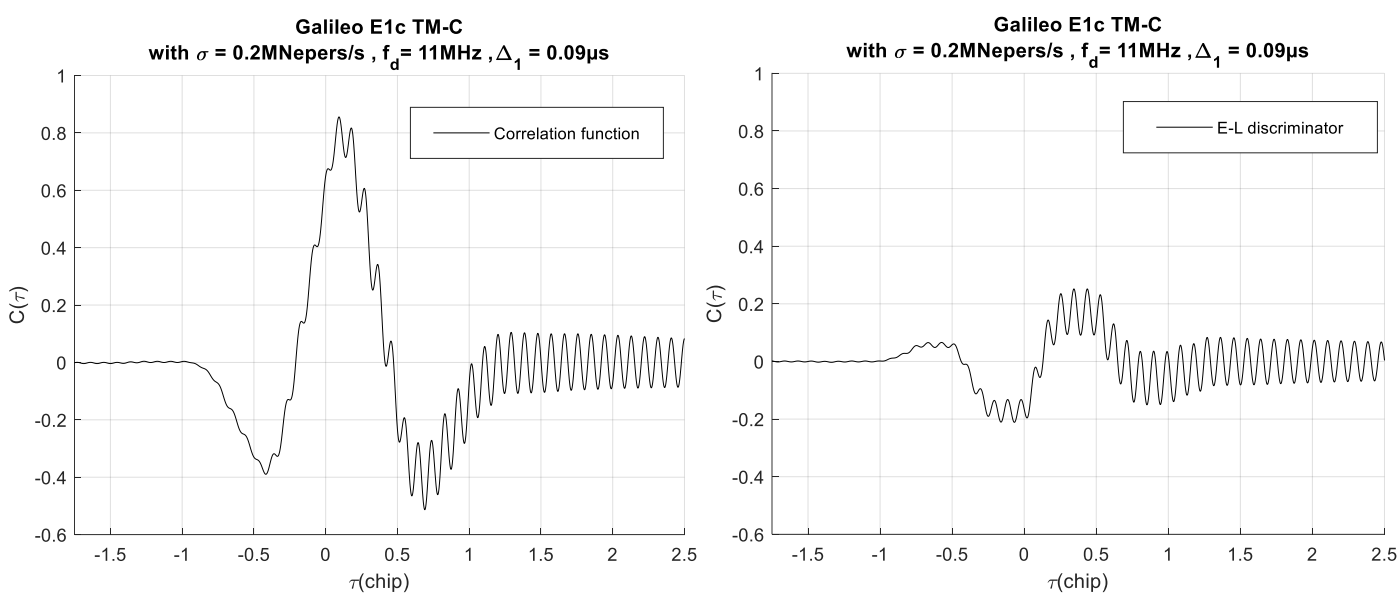

Figure 1. Example of correlation with multiple peaks (left) and the associated discriminator E-L with multiple zero crossings (right) for Galileo E1c signal distorted by TM-C with $\sigma=0.2$ Mnepers, $F_{d}=11 \mathrm{MHz}$ and $\Delta=0.09 \mu \mathrm{s}$

When dealing with such heavily distorted correlation peaks, the tracking loop behavior is uncertain. However, it is certain that in this situation, the real receiver tracking loop behavior will be driven by two main parameters:

- the tracking loop pull-in area: it corresponds to the area of the correlation function where the tracking loop will search for a peak and converge towards it.

- the distorted peak amplitude: if the distortion creates a peak that is much lower than the nominal one, this will result in a decrease of the estimated signal to noise density ratio (C/NO), a degradation of the tracking accuracy or even a loss of lock.

More details on the considered definition for these parameters in this study are given in the next section.

Here is a tentative description of the chain of events following the occurrence of an EWF.

1. the correlation peak that was tracked is suddenly shifted due to the correlation function offset induced by the EWF.

2. If the shift is in the pull-in area of the delay tracking loop, then, the loop may be able to continue its tracking process.

3. If the shift is larger than the tracking loop pull-in area, then the loop is not able to have a sufficiently stable tracking of the distorted signal, and the tracking loop begins to diverge. The C/NO estimated by the tracking loop, based on the tracked peak amplitude, will decrease and can trigger a loss of lock.

4. The tracking process may be stopped and a re-acquisition process could begin.

5. If so, the reacquired peak will likely be the one that has the highest amplitude as it is required by REQ-166 ("The equipment shall acquire the main correlation peak for each Galileo ranging source used in the navigation solution") in ED259 [5], not particularly the one closest to the nominal peak.

Figure 2 represents the diagram of the chain of event expected at the receiver.

As can be seen, different possible receiver behaviors can occur after the occurrence of an EWF, and many depend on some design choices of the receiver. This situation may be further complicated by the implementation of peak ambiguity resolution techniques for $\mathrm{BOC}$ modulations or multipath mitigation techniques [6]-[8], that may react to the presence of multiple peaks or correlation function distortion. 
In this paper, we have voluntarily chosen to model the receiver behavior with a simplified model and without taking into account complex tracking techniques related to multiple BOC correlation peak or multipath mitigation.

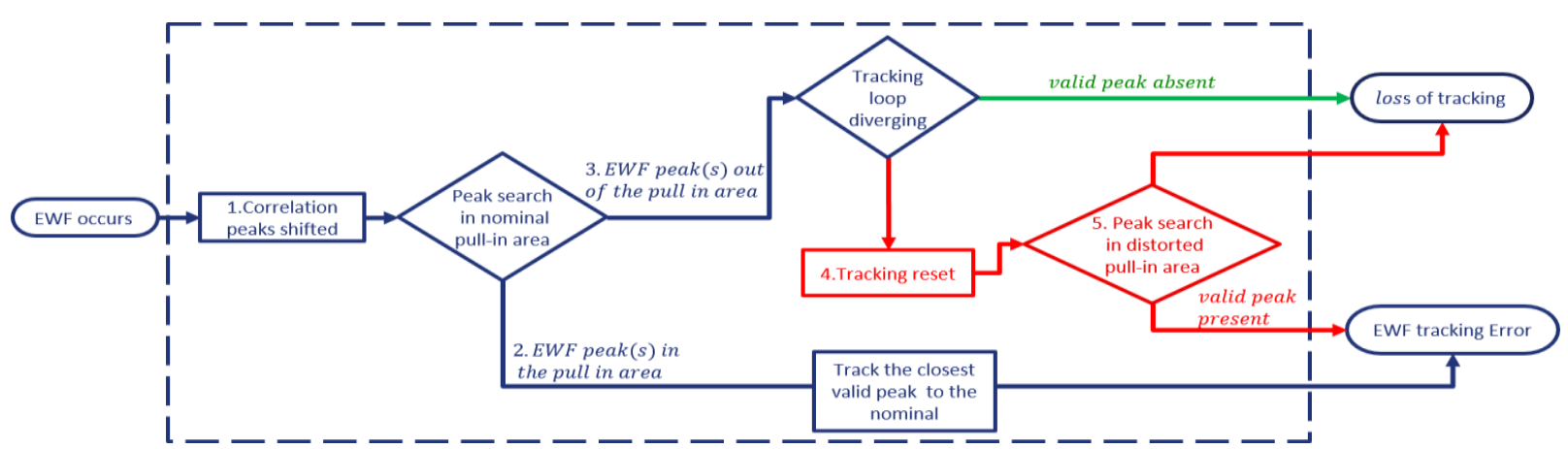

Figure 2. Expected chain of event in the GNSS receiver following EWF occurrence.

\section{SECTION 3 - MODELS OF THE RECEIVER BEHAVIOR IN PRESENCE OF MULTIPLE CORRELATION PEAKS}

In this study, two models of receiver behavior are considered:

- $\quad$ RBM 1: Model for receiver behavior with uninterrupted tracking

- $\quad$ RBM 2: Model for receiver behavior with loss of tracking and re-acquisition process (including steps 4 and 5 depicted in red in Figure 2). It also corresponds to the first acquisition applied when the EWF occurs before the satellite starts to be visible to the user or reference station receiver (called rising satellite case).

The overall process to determine the position of the correlation peak in presence of potential multiple zero crossing is described in Figure 3.

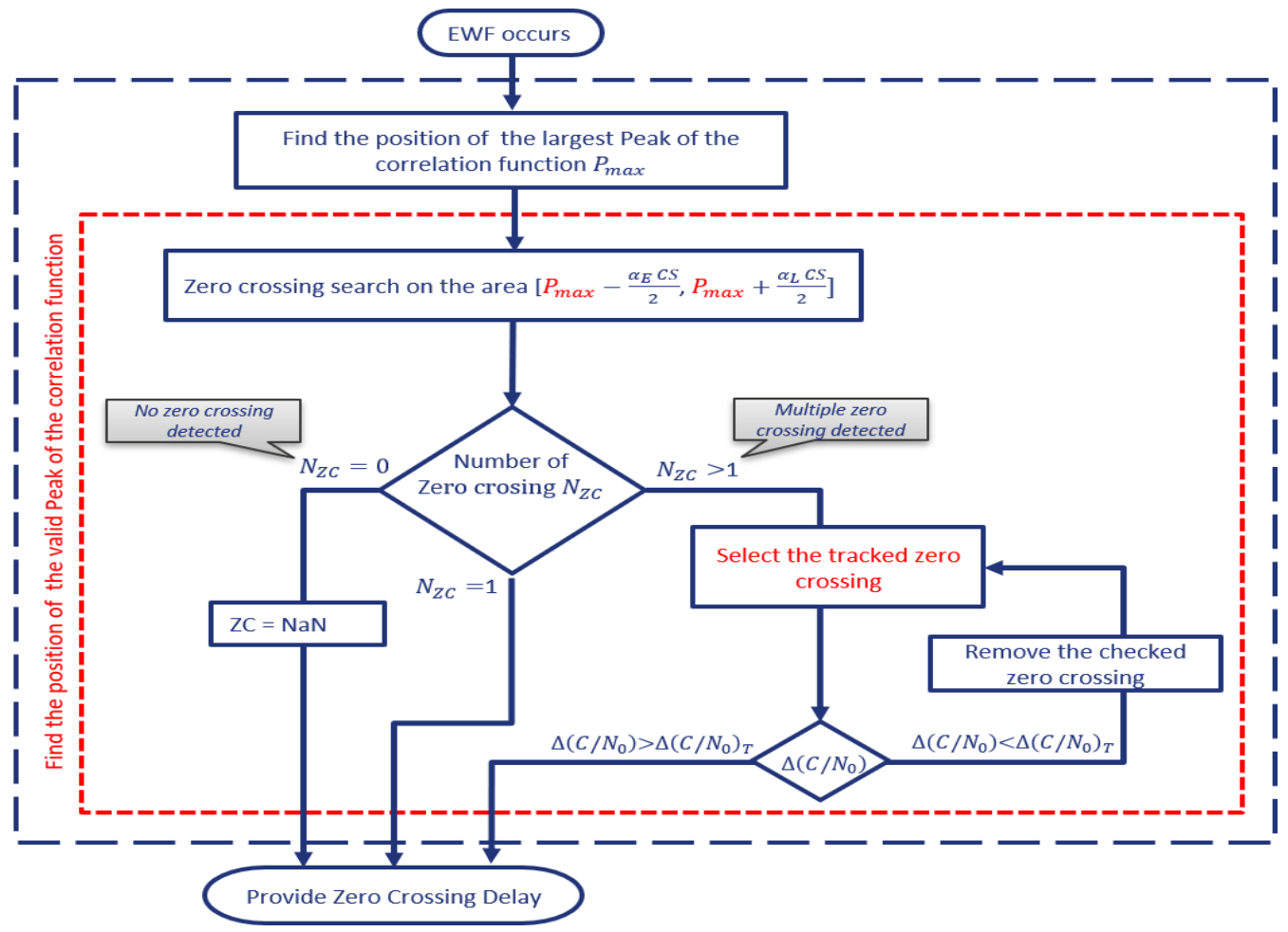

Figure 3. Zero crossing research diagram for Model 1 \& 2 
For both models, the tracking loop pull-in area $A_{E-L}$ on the E-L discriminator function [9] has to be defined for each receiver parameters of the User Space. In this study, the pull-in area is defined as the discriminator area with positive slope around the zero-crossing position obtained in the nominal condition (when no EWF is applied) taking into account only the RF/IF filtering effect. It is defined as:

$$
A_{E-L}=\left[P_{\max }-\frac{\alpha_{E} C S}{2}, P_{\max }+\frac{\alpha_{L} C S}{2}\right](\text { chips })
$$

Where

- $\quad \alpha_{E}$ and $\alpha_{L}$ are factors computed for each tested user configuration to define the stable area with a positive slope within the filtered discriminator function.

- $\quad P_{\max }$ is the position of the maximum peak that is determined:

○ on the distorted correlation function for RBM 2

0 on the nominal correlation function for RBM 1.

- $\quad C S$ is the chip spacing between the Early and Late replica

Figure 4 shows an example of the nominal Galileo E1c correlation functions and discriminators filtered by a $6^{\text {th }}$ order Butterworth filter with 12 and $24 \mathrm{MHz}$ bandwidths. The green areas on the discriminator function (and the red one on the correlation function) correspond to the pull-in areas obtained for each case of receiver configuration with an E/L chip spacing of 0.1 chip. It can be observed that the pull-in area width (proportional to $\alpha_{E}+\alpha_{L}$ ) varies a lot (by a factor larger than 2 in the example) according to the user parameters.
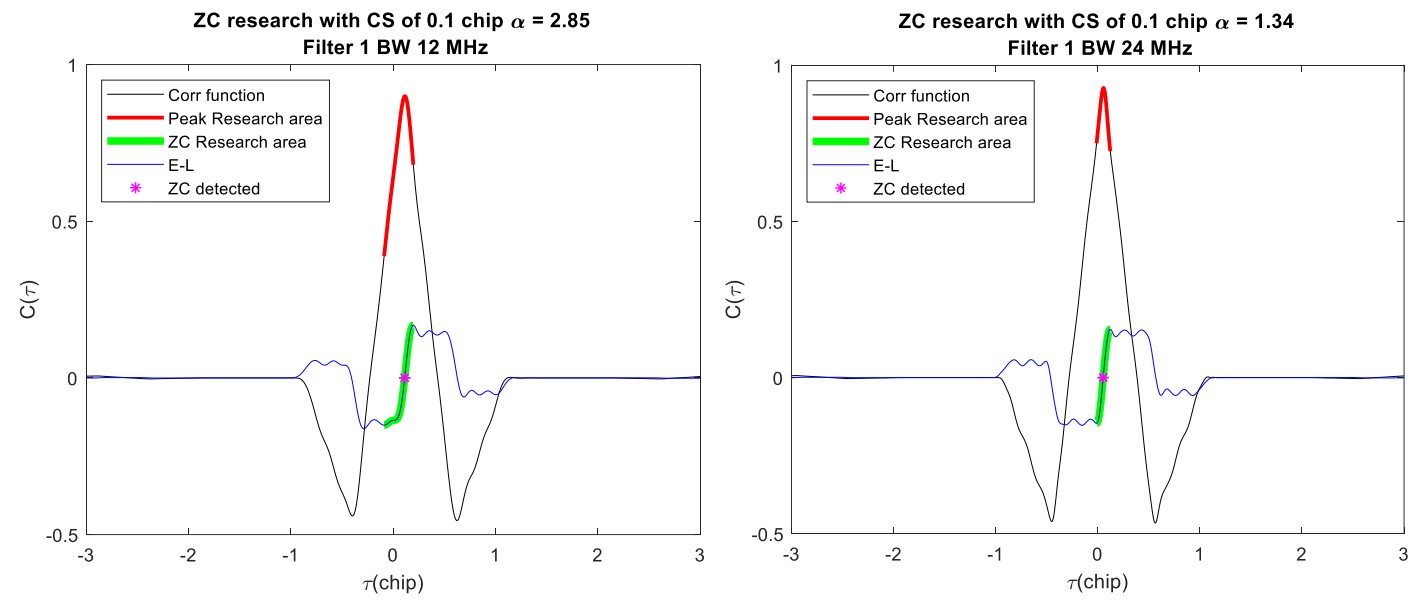

Figure 4. Examples of pull-in area defined for Galileo E1C E-L discriminator filtered by $6^{\text {th }}$ order Butterworth filter and bandwidth of 12 (Left) and 24 (right) $\mathrm{MHz}$ and an E/L spacing of 0.1 chip.

Depending on the number of zero crossings found in $A_{E-L}$, a different process is applied:

- if there is no zero-crossing detected, the distorted signal cannot be tracked, and the associated DLL delay is not taken into account.

- if only one zero-crossing is detected, the associated delay is saved.

- $\quad$ if more than one zero-crossings are detected, the closest one found when starting the research from $P_{\text {max }}$ and in the DLL direction search (right or left side of $P_{\text {max }}$, determined by the value of the discriminator after the introduction of the EWF) is selected. Depending on the tested RBM, the first selected peak corresponds to:

- the closest zero crossing to the nominal one for RBM 1

o the first stable zero crossing for RBM 2 
If the associated correlation peak to this zero crossing has a correlation loss lower than the correlation loss threshold $\Delta\left(C / N_{0}\right)_{T}$, the associated delay is saved as the DLL shift induced by the distortion. Otherwise, it is assumed that this correlation peak cannot be tracked by the DLL, and the next zero crossing is checked to compare its correlation peak amplitude loss to the threshold $\Delta\left(C / N_{0}\right)_{T}$. This process is repeated until reaching a valid zero crossing (with peak correlation loss lower than the correlation loss threshold $\left.\Delta\left(C / N_{0}\right)_{T}\right)$ or going though all detected zero crossings within the pull-in area.

The considered threshold on the $C / N_{0}$ loss, noted $\Delta\left(C / N_{0}\right)_{T}$, is computed as:

$$
\Delta\left(C / N_{0}\right)_{T}=\left(C / N_{0}\right)_{\max }-\left(\left(C / N_{0}\right)_{\text {tracking }}^{\text {threshold }}-\delta\left(C / N_{0}\right)_{\text {receiver }}\right)+\delta \Delta\left(C / N_{0}\right)
$$

Where

- $\delta\left(C / N_{0}\right)_{\text {receiver }}$ is the margin taken on the minimum $C / N_{0}$ tracked by the receiver. It is set to $2 \mathrm{~dB}$.

- $\delta \Delta\left(C / N_{0}\right)$ is the uncertainty margin taken on $\Delta C / N_{0}$. It is set to $2 \mathrm{~dB}$.

- $\left(C / N_{0}\right)_{\max }$ is the maximum $C / N_{0}$ received at the user receiver. It is computed as:

$$
\left(C / N_{0}\right)_{\max }=P_{\max }+G_{\text {ant }, \max }-C_{\text {loss } \min }-N_{0}
$$

- Maximal signal power $P_{\max }$ received at the receiver front end

- For DFMC SBAS user, only maximal signal power at the pilot channel is considered as given in Table 4 by $P_{\max }^{\text {pilot }}$.

- Maximum antenna gain $G_{\text {ant,max }}: 4 \mathrm{~dB}$ for L1 \& L5 [10].

- Minimum correlation loss $C_{\text {loss,min }}: 1 \mathrm{~dB}$ for wide band signals and receiver band larger than 24 $\mathrm{MHz}[11]$.

- Noise power density $N_{0}$.

- $\left(C / N_{0}\right)_{\text {tracking }}^{\text {threshold }}$ is the minimum $\mathrm{C} / \mathrm{NO}$ tracked by the receiver.

The applied threshold $\Delta\left(C / N_{0}\right)_{T}$ for GPS L1 and L5Q and Galileo E1 and E5a are given in Table 4.

\begin{tabular}{|c|c|c|c|c|c|c|}
\hline Signal & $\begin{array}{c}P_{\max } \\
(\mathrm{dBW})\end{array}$ & $\begin{array}{l}P_{\max }^{\text {pilot }} \\
(\mathrm{dBW})\end{array}$ & $\begin{array}{c}N_{0} \\
\text { (dBW/Hz) }\end{array}$ & $\begin{array}{c}C / N_{0_{\text {max }}} \\
(\mathrm{dBHz})\end{array}$ & $\begin{array}{c}\left(C / N_{0}\right)_{\text {tracking }}^{\text {threshold }} \\
(\mathrm{dBHz})\end{array}$ & $\begin{array}{c}\Delta\left(C / N_{0}\right)_{T} \\
(\mathrm{~dB})\end{array}$ \\
\hline GPS L1 & $-153[12]$ & - & $-201.5[13]$ & 51.5 & $29[14]$ & 26.5 \\
\hline GPS L5Q & $-150[12]$ & -150 & $-200[15]$ & 53 & 24 [11], [15] & 33 \\
\hline Galileo $E 1(b+c)$ & $-151.45[16]$ & -154.45 & $-201.5[13]$ & 53.05 & 29 [17] & 28.1 \\
\hline Galileo E5a(I+Q) & $-149.45[16]$ & -152.45 & $-200[15]$ & 53.55 & 27 [11], [15] & 30.6 \\
\hline
\end{tabular}

Table $4 \triangle C / N_{0}$ threshold for GPS L1/L5Q and Galileo E1 and E5a

\section{SECTION 4 - OBSERVATION OF THE PRESENCE OF MULTIPLE CORRELATION PEAKS OVER THE EWF GALILEO E5A TS AND THE USER SPACE FOR THE TWO RECEIVER BEHAVIOR MODELS.}

In this section, the distribution of the observed zero-crossings situations (depending on the number of the zero crossings found in the pull-in area) and the tracking biases associated to the selected zero crossing are presented for both RBM 1 \& 2. Each sample of these distributions corresponds to a TM-A, B and C case processed by one tested receiver configuration (defined by the filter type, bandwidth and E/L chip spacing). Figure 5 and Figure 6 show the distribution of the number of obtained zero crossings (divided by the total number of simulated cases) with respect to the two tested receiver behavior models 1 \& 2 (RBM 1 \& 2) for TM-A, B and C applied to Galileo E5a signal. It appears that one zero crossing (unique zero crossing situation) is obtained for around $80 \%$ of the couples $\{$ TM-A, B and $C$ distortion / receiver configuration $\}$ for both RBM 1 \& 2. Among the remaining 20\%, most of $\{$ TM-A, B and C 
distortion / receiver configuration \} couples give a no-zero crossing situation and only few of them (less than $0.3 \%$ ) give multiple zero crossing situation for both RBM 1 \& 2.

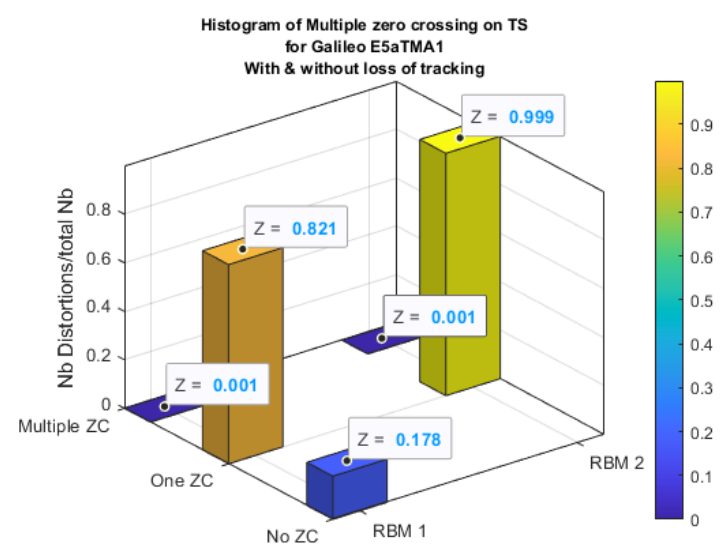

Figure 5. Distribution of the number of the couples TM-A cases and associated receiver configurations with respect to the number of zero crossing obtained with the two receiver models 1 \& 2
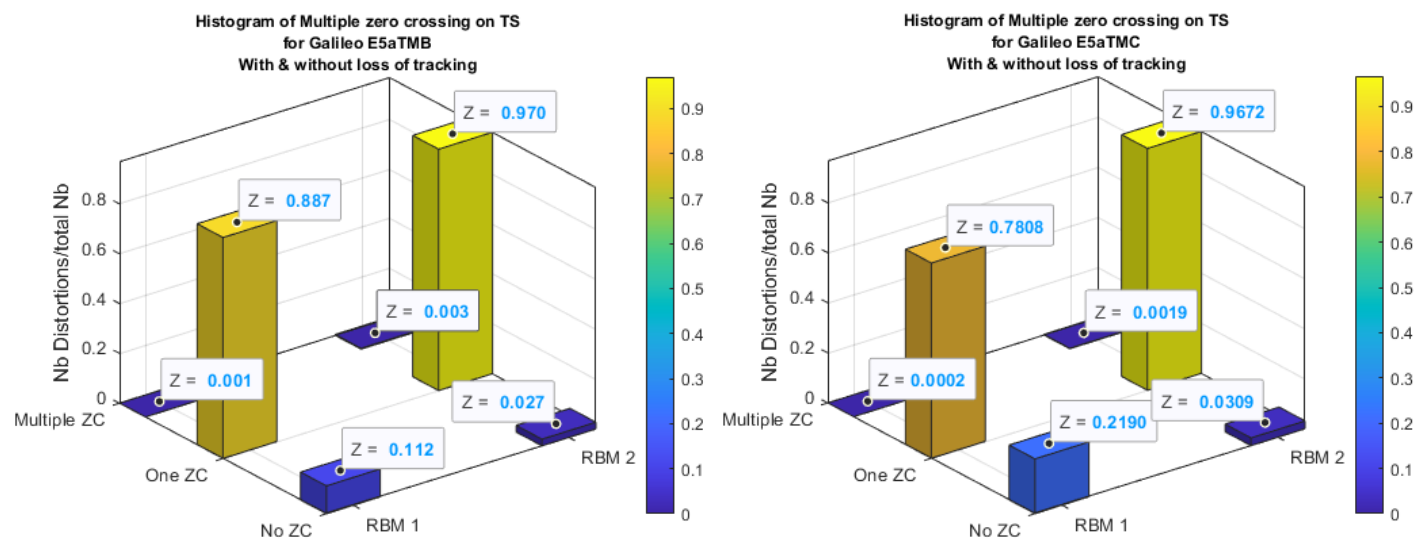

Figure 6. Distribution of the number of the couples TM-B (right) and TM-C (left) cases and associated receiver configurations with respect to the number of zero crossing obtained with the two receiver models 1 \& 2

The effect of each EWF is evaluated by the induced absolute tracking bias estimated as the difference between the observed delays on the distorted and nominal correlation function processed by the same receiver configuration. The absolute tracking bias difference between the bias obtained with RBM 1 and 2 for each TM-A, B and C and each tested receiver configuration is computed as:

$$
\delta b_{i}=\left|b_{-} u s e r_{\mathrm{RBM} 1}^{i}-b_{-} u s e r_{\mathrm{RBM} 2}^{i}\right|
$$

Where $b_{-} u s e r_{\mathrm{RBM} 1}^{i}$ and $b_{-} u s e r_{\mathrm{RBM} 2}^{i}$ are the absolute tracking bias obtained with user receiver configuration $i$ (defined in the User Space of Table 3) with RBM 1 and 2 respectively. A large $\delta b_{i}$ indicates a case where the EWF can potentially lead to an integrity risk for one user and potentially not for the other user. It is therefore an indicator of the sensitivity of an EWF impact to the RBM.

The distribution of the absolute bias difference $\delta b_{i}$ is given in Figure 7 and Figure 8 for TM-A, B and C. It shows that most of the absolute bias differences are around zero except very few TM-B and C cases where the difference $\delta b_{i}$ 
can go up to 85 meters. It means that for most couples \{distortion / receiver configuration\}, the same peak is tracked in RBM 1 and 2. However, for less than 3\% of couples (TM-B and C distortion / receiver configuration), two different peaks (separated by 10 to 80 meters) are tracked with RBM 1 and 2 .

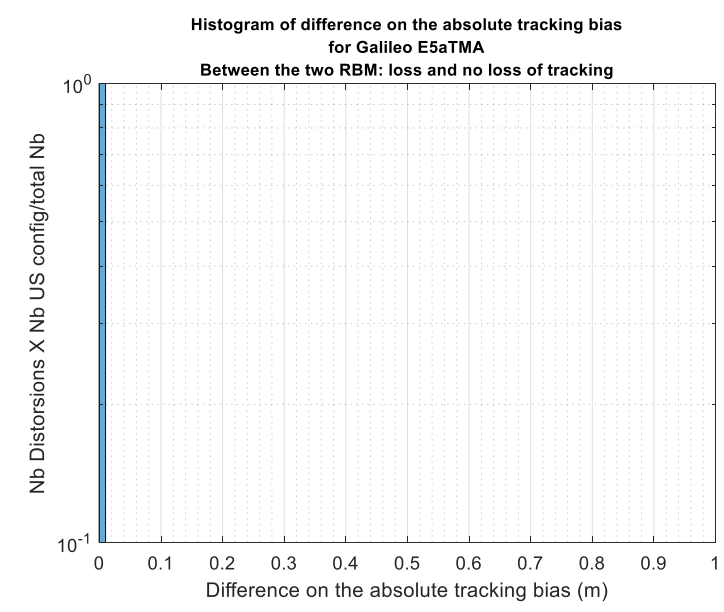

Figure 7. Distribution of the difference on the absolute tracking bias obtained with TM-A and the tested user receiver configuration obtained with the two receiver models 1 \& 2
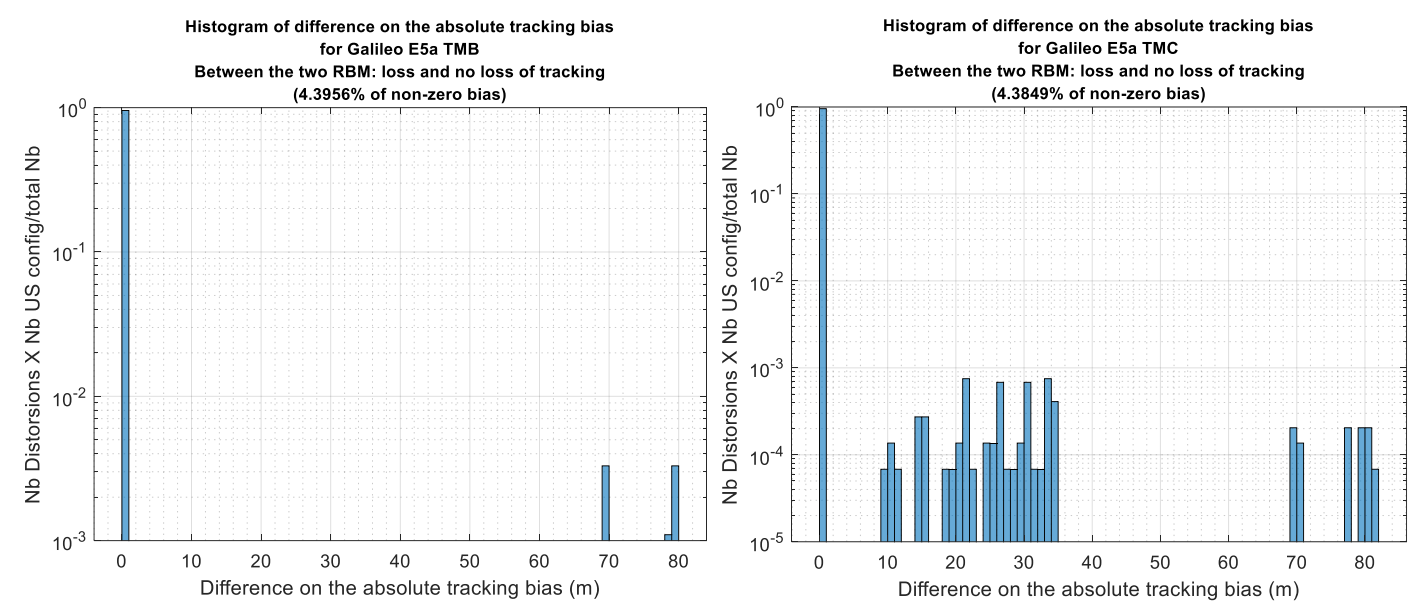

Figure 8. Distribution of the difference on the absolute tracking bias obtained with TM-B (left) and TM-C (right) and the tested user receiver configuration obtained with the two receiver models $1 \& 2$

\section{SECTION 5 - ANALYSIS OF THE IMPACT OF THE RECEIVER BEHAVIOR MODEL ON THE TRACKING ERROR:}

\section{5.a. Sensitivity of the Tracking Error obtained from both RBMs to the Threat Space}

The question addressed in this section is to determine in which area of the TM-A, B and C TSs, Multiple Peaks situations are more likely to appear and may induce different tracking bias depending on the tested RBM.

Figure 9 and Figure 10 present the distribution of the number of receiver configurations inducing multiple zero crossings (divided by the total number of simulated receiver configurations) with respect to TM-B parameters $\left(\mathrm{F}_{\mathrm{d}}\right.$ and $\sigma$ ) for TM-B and TM-C with $\Delta= \pm 0.12 \mu$ s applied to Galileo E5a. To illustrate the observed results for TM-C distortions, only one example of $|\Delta|$ value is given in this section and in the following ones. It corresponds to the TM- 
C cases where multiple zero crossings situation is observed more often in the TS (compared to the situation observed with the other values of $|\Delta|$ ). This example is sufficient to demonstrate that the multiple zero crossing situation can occur for TM-C with at least one value of $\Delta$.

For TM-B, it can be observed that with RBM1 (left figure), the multiple zero crossing situation is encountered in a specific part of the TS $\left(\mathrm{F}_{\mathrm{d}} \in[0.3 ; 1] \mathrm{MHz}\right.$ and $\sigma \in[0.3 ; 3]$ Mnepers/s). For RBM2 (right figure), the multiple zero crossing situation affects a wider area of the TS. For TM-C (with $\Delta= \pm 0.12 \mu \mathrm{s}$ ) almost the same TS area is encountering multiple zero crossing situations with RBM $1 \& 2$ (except the tiny area around very low $\sigma$ and $F_{d}$ and the area around $\mathrm{F}_{\mathrm{d}}=0.2 \mathrm{MHz}$ ).

However, for both TM-B and TM-C, more receiver configurations are inducing multiple zero crossing with RBM2 (uninterrupted tracking). For RBM1, less than 15\% of receiver configurations are in multiple zero crossing situation (for the concerned distortions) and it reaches 30\% for RBM2.
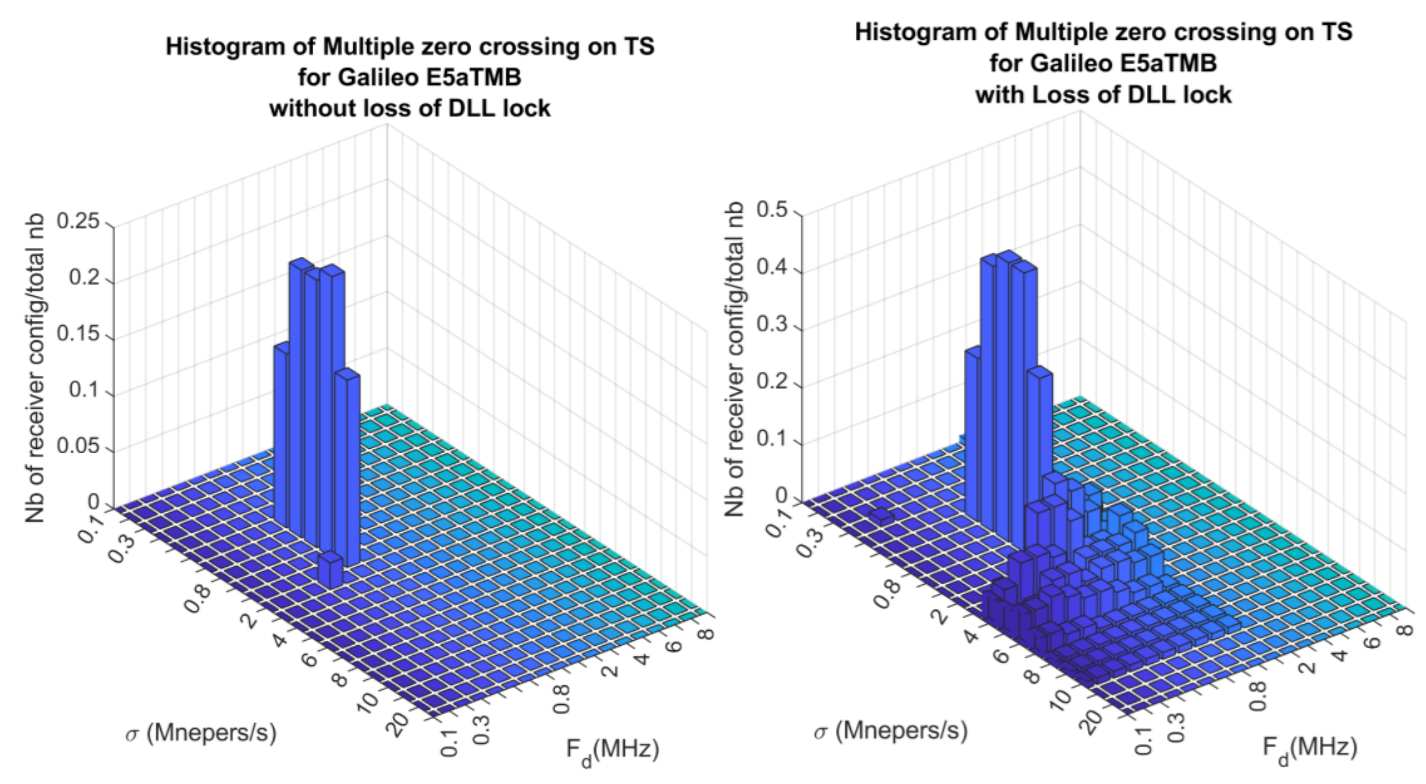

Figure 9. Distribution of the number of receiver configurations inducing multiple zero crossing with respect to TM-B parameters for the two RBM 1 (left) \& 2 (right) for TM-B

Figure 11 represents the number (divided by the total number of tested receiver configurations) of receiver configurations giving a difference on the absolute bias $\delta b_{i}$ larger than $1 \mathrm{~cm}$ with respect to $\sigma$ and $\mathrm{F}_{\mathrm{d}}$ parameters for TM-B and TM-C (with $\Delta= \pm 0.12 \mu s$ ). It shows the TS area where different peaks (located at a distance larger than 1 $\mathrm{cm}$ ) are tracked with RBM 1 and 2. It appears that the TS areas experiencing multiple zero crossing situation in the pull-in area with RBM 1 \& 2 (shown in Figure 9 and Figure 10) are not the one where different peaks are tracked with RBM 1 \& 2. The observation of two distinct RBM tracking a different peak can be due to two origins:

a. If the same (or almost) pull-in area is considered for RBM1 and RBM2 and the EWF induces multiple peaks in this pull-in area, each RBM can track a different peak

b. the EWF causes a loss of tracking lock for RBM 2, which triggers a re-acquisition. The re-acquired peak is then searched at a different location from RBM 1. In this case the pull in areas considered can be distinct since they are centered around $P_{\text {max }}$ that is potentially different between RBM 1 and 2 . 

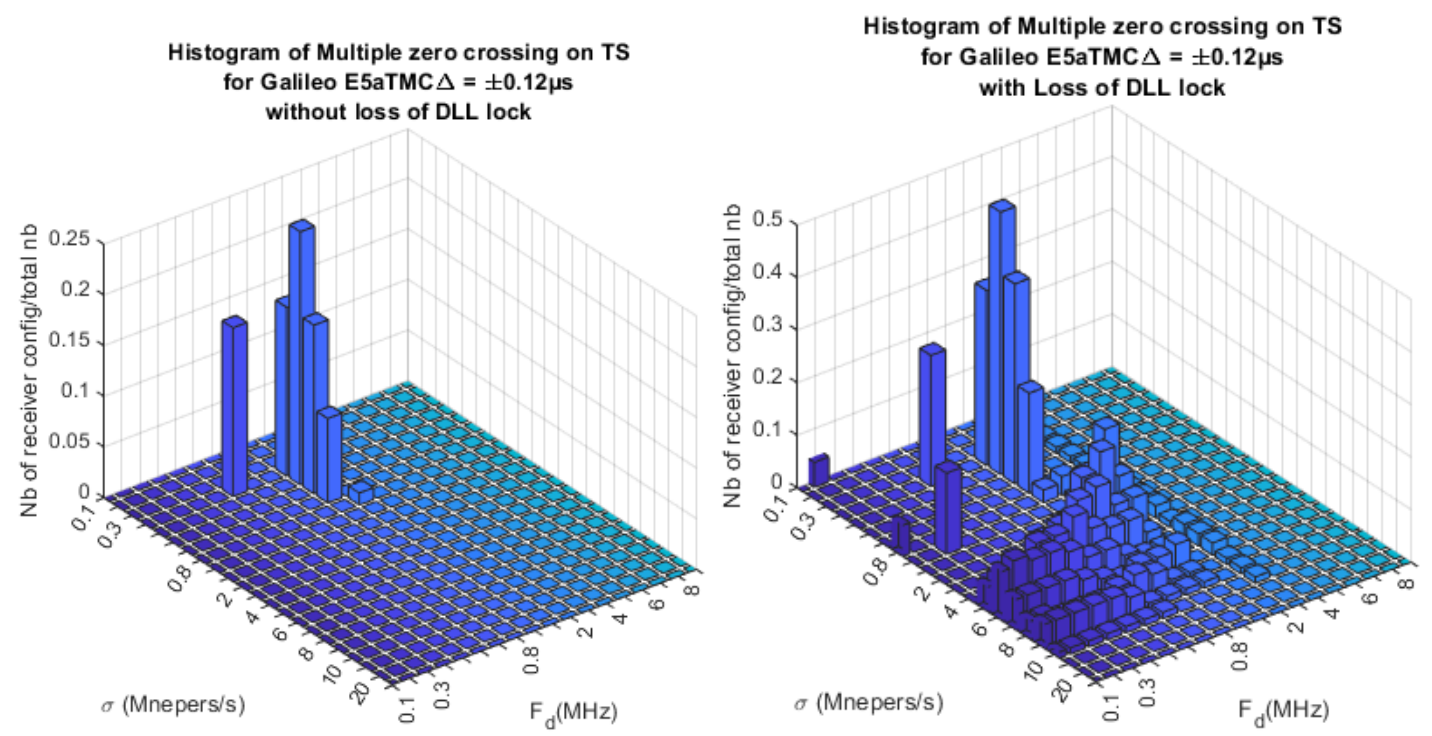

Figure 10. Distribution of the number of receiver configurations inducing multiple zero crossing with respect to TM-B parameters for the two RBM 1 (left) \& 2 (right) for TM-C with $\Delta= \pm 0.12 \mu \mathrm{s}$

The analysis of Figure 11 shows that a subset of receiver configurations gives $\delta b_{i}>1 \mathrm{~cm}$ for a different TS area from the one inducing multiple zero crossings. This shows that the second origin (b) described above is the cause of the large difference of absolute bias between the two considered RBMs. This observation confirms the importance and the impact of the notion of pull-in area when analyzing the multiple zero crossing situation.

For TM-B, less than $20 \%$ of the tested receiver configurations give different tracked peak in RBM 1 \& 2 . However, for TM-C, this percentage goes up to $24 \%$.
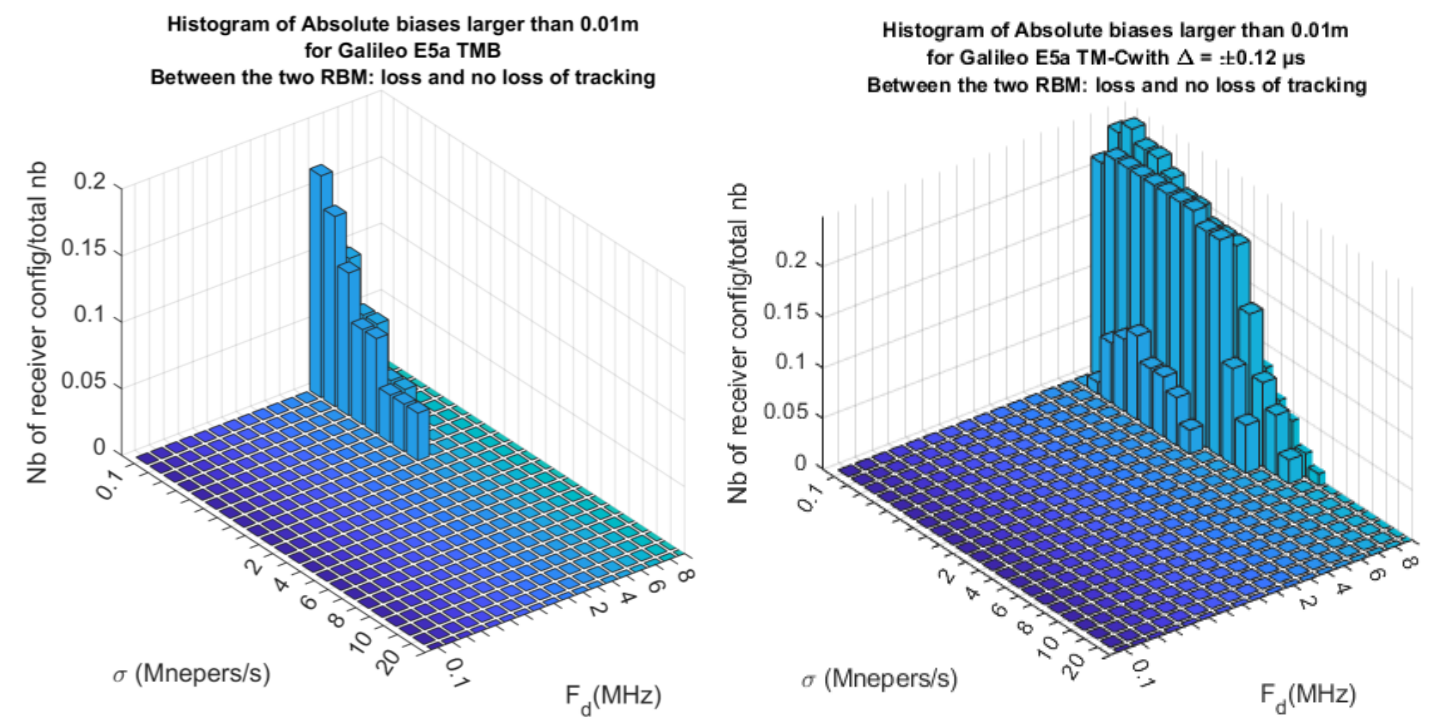

Figure 11. Distribution of the number of receiver configurations inducing a difference larger than $1 \mathrm{~cm}$ on the absolute tracked peaks biases obtained with RBM 1 \& 2 with respect to $T M-B$ parameters for TM-B (left) and TM-C with $\Delta= \pm 0.12 \mu s$ (right)

\section{5.b Sensitivity of the Tracking Error induced in the multiple zero crossing situation to the User Space}


The question addressed in this section is which User Space parameters are more likely to experience Multiple Peaks situation that induces different tracking bias depending on the tested RBM. They correspond to the 15 or $30 \%$ of receiver configuration experiencing multiple zero crossing situation for some area of TM-B and TM-C TS as defined in section 5.a.

The distribution of the TM-B and TM-C cases inducing multiple zero crossing (divided by the total number of distortions) on Galileo E5a with respect to tested filter bandwidths and chip spacings is analyzed. Contrary to the Threat Space, it seems that all the user space is affected by multiple zero crossing situations. Hence, the 15 or $30 \%$ of receiver configurations inducing multiple zero crossing can take any value among the tested filter bandwidths and E/L chip spacings.

Figure 12 shows the distribution of the number of TM-B and TM-C, that induce two different tracked peaks when applying RBM 1 and 2 with a tracking bias difference $\delta b_{i}$ larger than $1 \mathrm{~cm}$, with respect to the filter bandwidth and chip spacing (of Table 3). It appears that only some of the receiver configurations give two different peaks in RBM 1 $\& 2$ with an associated bias difference larger than $1 \mathrm{~cm}$. For example, with an E/L chip spacing of 1 chip and all filter bandwidths (from 12 to $22 \mathrm{MHz}$ ), the same correlation peak is always tracked with RBM 1 \& 2 (since separated with less than $1 \mathrm{~cm}$ ) for TM-B and TM-C (with $\Delta= \pm 0.12 \mu \mathrm{s}$ ).

Then, even if all tested receiver configurations can induce multiple zero crossing situation with some TM-B and C cases (for both RBM 1\&2), only some of them can give different tracked peaks when applying RBM $1 \& 2$.
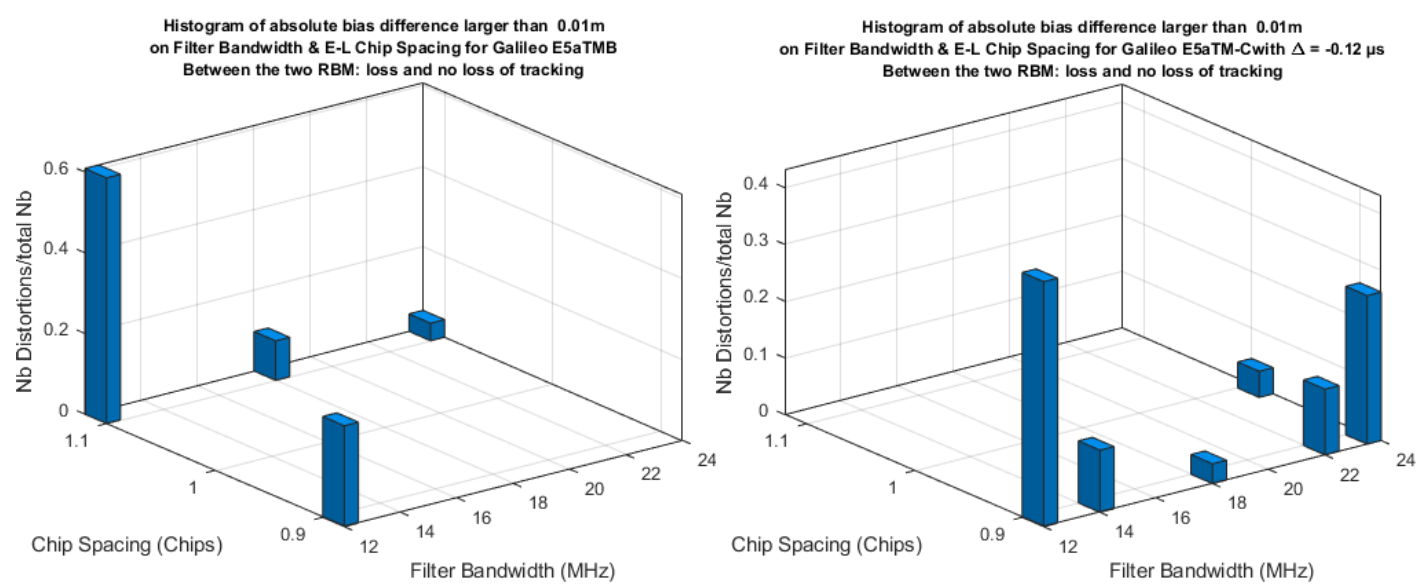

Figure 12. Distribution of the number of receiver configurations inducing a difference larger than $1 \mathrm{~cm}$ on the absolute tracked peaks biases obtained with RBM 1 \& 2 with respect to US parameters: E/L chip spacing and filter bandwidths for TM-B (left) and TM-C with $\Delta=-0.12 \mu s$ (right).

\section{SECTION 6 - CONCLUSION}

In this paper, only Galileo E5a results are presented to illustrate the observed impact of RBM1 and 2 and the multiple zero crossing induced by EWF situation. The results obtained for TM-A, B and C are summarized in Table 5 and Table 6. Table 5 gives the percentage of multiple zero crossing situations observed in the pull-in area for RBM 1 and 2 and Table 6 shows the maximum absolute difference $\delta b_{i}$ and the percentage of non-zero values for each TM.

Table 5. Percentage of the couples TM (-A, B and C) and receiver configurations inducing multiple zero crossing in the pull in area for RBM 1 \& 2 for Galileo E5a

\begin{tabular}{|c|c|c|c|c|}
\hline \multicolumn{2}{|c|}{ ICAO TM } & TM-A & TM-B & TM-C \\
\hline \multirow{2}{*}{$\begin{array}{c}\text { Galileo E5a } \\
\text { DFMC }\end{array}$} & RBM 1 & $0.1 \%$ & $0.1 \%$ & $0.19 \%$ \\
\cline { 2 - 5 } & RBM 2 & $0.1 \%$ & $0.3 \%$ & $0.02 \%$ \\
\hline
\end{tabular}


Table 6. Maximum $\delta b_{i}$ and percentage of $\delta b_{i} \neq 0$ obtained for TM-A, B and C for Galileo E5a

\begin{tabular}{|c|c|c|c|c|}
\hline \multicolumn{2}{|c|}{ ICAO TM } & TM-A & TM-B & TM-C \\
\hline \multirow{2}{*}{$\begin{array}{c}\text { Galileo E5a } \\
\text { DFMC }\end{array}$} & $\operatorname{Max}\left(\delta b_{i}\right)$ & $0 \mathrm{~m}$ & $80 \mathrm{~m}$ & $82 \mathrm{~m}$ \\
\cline { 2 - 5 } & $\%$ age of $\delta b_{i} \neq 0$ & $0 \%$ & $4.39 \%$ & $4.38 \%$ \\
\hline
\end{tabular}

The presented results for the assessment of multiple zero crossings observed in pull-in area with RBM 1 (uninterrupted tracking) and 2 (reacquisition in case of loss of tracking) for TM-A, B and C applied to Galileo E5a signal show that only few EWF cases (less than $0.3 \%$ ) are concerned. These cases are located around low $\sigma$ and $F_{d}$ values for TM-B and TM-C. However, all tested User Space receiver configurations can induce this situation with multiple zero crossing in the pull-in area when applying both RBM $1 \& 2$.

The analysis of the effect of the tested receiver behaviors on the obtained tracking bias show that, for some TS area, two different peaks separated by a large shift (that can reach tens of meters for TM-C) are tracked with RBM 1 \& 2 . However, less than $5 \%$ ( $0 \%$ for TM-A) of couples \{receiver configuration / distortion\} induce two different peaks tracked by RMB1 and 2 and separated by a distance larger than $1 \mathrm{~cm}$. It is then important to define the possible real receiver behaviors in presence of EWF in order to evaluate the worst (highest value inducing the lowest and most demanding $\mathrm{P}_{\mathrm{md}}$ requirement) absolute and differential bias induced by the TM-A, B and $\mathrm{C}$. The computation of the worst induced absolute or differential bias is needed to characterize the effect of each distortion in order to evaluate the integrity risk that may be caused by its presence (through the evaluation of the associated missed detection probability $\mathrm{P}_{\mathrm{md}}$ that needs to be respected).

The tested receiver behavior RBM 1 \& 2 correspond to two extreme receiver reactions to EWF occurrence. Different receiver behaviors (from RBM $1 \& 2$ ) may give different - and possibly worst (, ie with lower associated $P_{m d}$ ) - results for EWF induced tracking bias. In order to have a more accurate simulation of the receiver behavior in presence of EWF and the tracked peak in multiple zero crossing situation, one solution would be to agree on the main peak definition for this scenario.

Another solution would be to test a real receiver behavior in presence of simulated EWF generated by an RF signal simulator. However, the obtained results would only reflect the behavior of the tested receiver and cannot be generalized to all user receivers.

Future work on the evaluation of the multipath and noise effect on the receiver behavior in presence of EWF would be necessary to carry out more accurate analysis of the receiver reaction in presence of EWF with multiple zero crossing situation. In addition, the considered assumptions to define the pull-in area need to be further consolidated for a more realistic analysis of multiple zero crossings issue.

\section{Acknowledgement}

The authors would like to thank Mikael Mabilleau (EUSPA: European Union Agency for the Space Program) and Denis Bouvet (Thales AVS France) for providing relevant feedback on the study presented in this paper.

\section{References}

[1] P. K. Enge, E. Phelts, and A. M. Mitelman, "Detecting Anomalous Signals from GPS Satellites," 1999.

[2] ICAO, Annex 10 - Aeronautical Telecommunications, Volume 1 - Radio Navigation Aids, Sixth Edition., vol. 1. 2006.

[3] I. Selmi and M. Mabilleau, "SBAS message table changes linked to spare definition," presented at the Navigation System Panel (NSP) joint working groups - fifth meeting, Jan. 2020. 
[4] J. Rife and R. E. Phelts, "Formulation of a time-varying maximum allowable error for ground-based augmentation systems," IEEE Transactions on Aerospace and Electronic Systems, vol. 44, no. 2, pp. 548-560, Apr. 2008, doi: 10.1109/TAES.2008.4560206.

[5] EUROCAE, "ED-259- Minimum Operational Performance Standards for Galileo - Global Positioning System Satellite-Based Augmentation System Airborne Equipment," ED-259, Feb. 2019. Accessed: Jun. 25, 2019.

[6] D. Margaria, E. Falletti, A. Bagnasco, F. Parizzi, and A. Torchi, "A New Strategy to Mitigate Side-Peak Lock Errors in Tracking Multi-Peak Code Correlation Functions," presented at the NAVITEC, Noordwijk, Netherlands, Dec. 2014.

[7] D. Gómez-Casco, J. A. Garcia-Molina, A. Gusi-Amigó, M. Crisci, J. A. López-Salcedo, and G. Seco-Granados, "Mitigation of false locks in the acquisition of high-order BOC signals in HS-GNSS receivers," in 2016 International Conference on Localization and GNSS (ICL-GNSS), 2016, pp. 1-6.

[8] O. Julien, E. Cannon, G. Lachapelle, C. Mongrédien, and C. Macabiau, "A New Unambiguous BOC(n,n) Signal Tracking Technique," presented at the ENC GNSS, Rotterdam, Netherlands, May 2004. Accessed: Aug. 02, 2016.

[9] E. D. Kaplan and C. Hegarty, Eds., "Satellite Signal Acquisition, Tracking, and Data Demodulation," in Understanding GPS/GNSS: principles and applications, Third edition., Boston ; London: Artech House, 2017.

[10] "ICAO NSP JWGs/6-Flimsy 13." Jun. 08, 2020.

[11] G. Novella, A. Garcia-Pena, and C. Macabiau, "GNSS interference thresholds for L1 and L5 bands," v2.4, Nov. 2020.

[12] “GPS SARPS JWGs/6-Flimsy 21." GPS SARPS JWGs/6-Flimsy 21, Jun. 08, 2020.

[13] RTCA, "DO 235B - Assessment of Radio frequency Interference relevant to the GNSS L1 frequency band." Mar. $13,2008$.

[14] RTCA, "DO 229E - Minimum Operational Performance Standards (MOPS) for GPS/WAAS Airborne Equipment." Dec. 2016.

[15] RTCA, "DO 292 - Assessment of Radio Frequency Interference Relevant to the GNSS L5/E5A Frequency Band." Jul. 29, 2004.

[16] "ICAO NSP JWGs/6-Flimsy 23." Jun. 15, 2020.

[17] O. Julien, J.-L. Issler, and L. Ries, "Investigation of Galileo E1 OS/SoL Acquisition, Tracking and Data Demodulation Thresholds for Civil Aviation," presented at the ION GNSS, Portland, Oregon, Sep. 2011. 